\title{
Kinetic Isotope Effects as a Probe for the Protonolysis Mechanism of Alkylmetal Complexes: VTST/MT Calculations Based on DFT Potential Energy Surfaces
}

Binh Khanh Mai and Yongho Kim*

Department of Applied Chemistry and Institute of Natural Sciences, Kyung Hee University, 1 Seochun-Dong, Giheung-Gu, Yongin-Si, Gyeonggi-Do, 446-701, Korea

E-mail: yhkim@khu.ac.kr

Table S1. Mean unsigned errors (MUEs) for some geometric parameters of (COD) $\mathrm{Pt}^{\mathrm{II}} \mathrm{Me}_{2}$ with respect to experimental values $(\AA)$.

\begin{tabular}{llllllll}
\hline Method & ${ }^{1} \mathbf{P t}-{ }^{2} \mathbf{C}$ & ${ }^{1} \mathbf{P t}-{ }^{3} \mathbf{C}$ & ${ }^{1} \mathbf{P t}-{ }^{4} \mathbf{C}$ & ${ }^{1} \mathbf{P t}-{ }^{5} \mathbf{C}$ & ${ }^{1} \mathbf{P t}-{ }^{8} \mathbf{C}$ & ${ }^{1} \mathbf{P t}-{ }^{9} \mathbf{C}$ & MUE \\
\hline B3LYP/D95V & 0.020 & 0.007 & 0.092 & 0.131 & 0.098 & 0.116 & 0.077 \\
B3LYP/6-31G(d,p) & 0.017 & 0.004 & 0.087 & 0.127 & 0.093 & 0.112 & 0.073 \\
M06-L/D95V & 0.019 & 0.006 & 0.073 & 0.104 & 0.079 & 0.089 & 0.062 \\
M06-L/6-31G(d,p) & 0.017 & 0.004 & 0.061 & 0.092 & 0.067 & 0.077 & 0.053 \\
M06/D95V & 0.018 & 0.005 & 0.069 & 0.102 & 0.075 & 0.087 & 0.059 \\
M06/6-31G(d,p) & 0.014 & 0.001 & 0.058 & 0.092 & 0.064 & 0.077 & 0.051 \\
BP86/LANL2TZ(f)/ & 0.015 & 0.002 & 0.008 & 0.035 & -0.150 & 0.020 & 0.038 \\
6-31+G(d) ${ }^{1}$ & 2.056 & 2.069 & 2.227 & 2.231 & 2.221 & 2.246 & \\
Exp. $^{2}$ & & & & & & & \\
\hline
\end{tabular}

Table S2. Selected bond distances $(\AA)$ between metal and ligands for stationary points of the protonolysis of (COD)Pt ${ }^{\mathrm{II}} \mathrm{Me}_{2}$ by TFA at the M06/6-31G(d,p) level.

\begin{tabular}{llllll}
\hline & ${ }^{1} \mathbf{P t}-{ }^{3} \mathbf{C}$ & ${ }^{1} \mathbf{P t}-{ }^{4} \mathbf{C}$ & ${ }^{1} \mathbf{P t}-{ }^{5} \mathbf{C}$ & ${ }^{\mathbf{1}} \mathbf{P t}-{ }^{8} \mathbf{C}$ & ${ }^{1} \mathbf{P t}-{ }^{9} \mathbf{C}$ \\
\hline $\mathbf{R}$ & 2.068 & 2.301 & 2.353 & 2.316 & 2.339 \\
$\mathbf{T S}_{\mathbf{d}}$ & 2.064 & 2.360 & 2.414 & 2.153 & 2.176 \\
$\mathbf{I}_{\mathbf{0 x}}$ & 2.066 & 2.482 & 2.513 & 2.491 & 2.562 \\
$\mathbf{T S}_{\mathbf{0 x}}$ & 2.067 & 2.420 & 2.448 & 2.273 & 2.282 \\
$\mathbf{P}$ & 2.052 & 2.357 & 2.421 & 2.140 & 2.153 \\
\hline
\end{tabular}


Table S3. Potential energies ( $\mathrm{kcal} / \mathrm{mol}$ ) of formation for reactant complex $\mathbf{R}$. The numbers in parenthesis include zero-point energies.

\begin{tabular}{lc}
\hline & Reactant Complex R \\
\hline B3LYP/D95V & $-11.4(-10.8)$ \\
B3LYP/6-31G(d,p) & $-5.55(-4.84)$ \\
M06-L/D95V & $-15.1(-14.8)$ \\
M06-L/6-31G(d,p) & $-9.99(-9.08)$ \\
M06/D95V & $-15.1(-13.9)$ \\
M06/6-31G(d,p) & $-8.55(-7.65)$ \\
IEFPCM/M06/6-31G(d,p) & $-5.96(-5.12)$ \\
SMD/M06/6-31G(d,p) & $-5.47(-4.40)$ \\
\hline
\end{tabular}




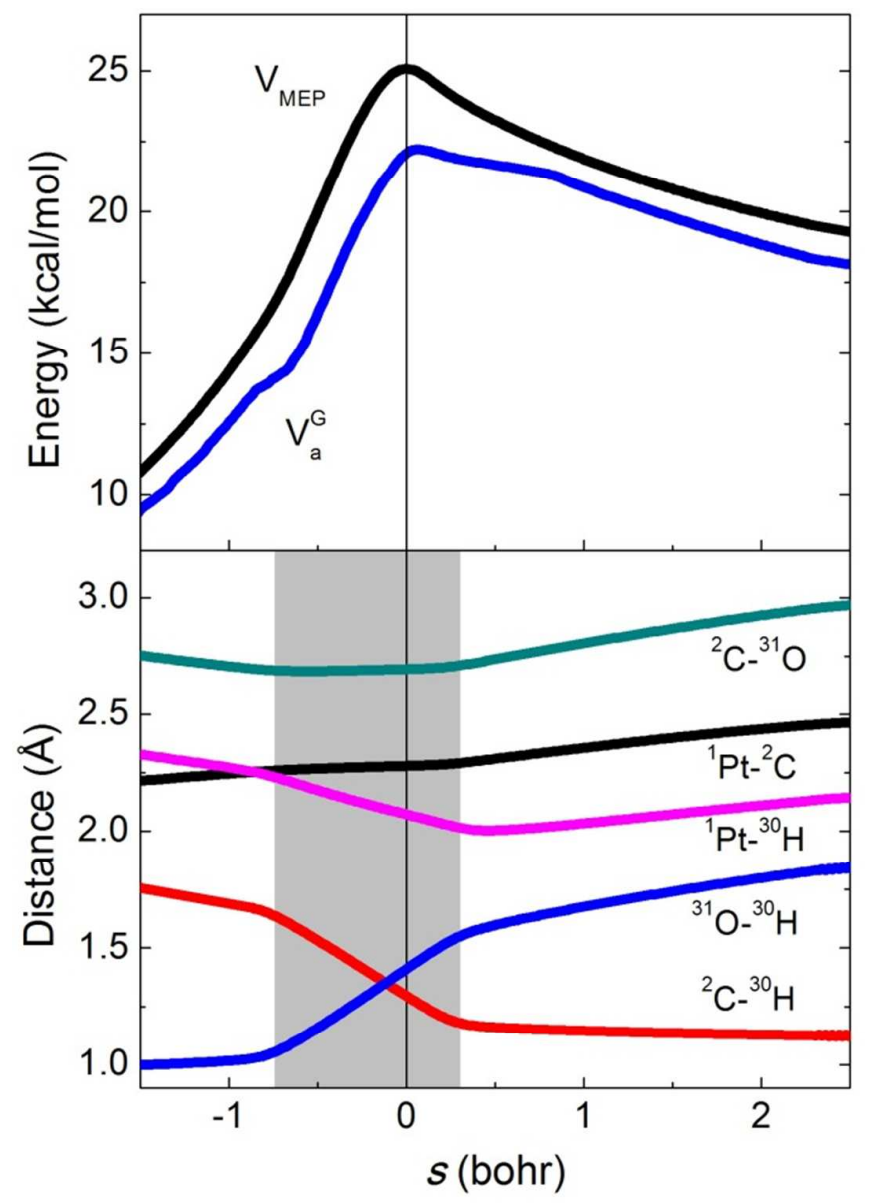

Figure S1. Potential ( $\left.V_{\mathrm{MEP}}\right)$ and adiabatic energy $\left(V_{\mathrm{a}}^{\mathrm{G}}\right)$ curves (black and blue, respectively) and bond lengths (as labeled) along the MEP of proton transfer in the protonolysis of (COD)Pt ${ }^{\mathrm{II}} \mathrm{Me}_{2}$ via concerted pathway at the M06/6-31G(d,p) level. The vertical line represents the position of the transition state $\mathbf{T S}_{\mathbf{d}}$. 


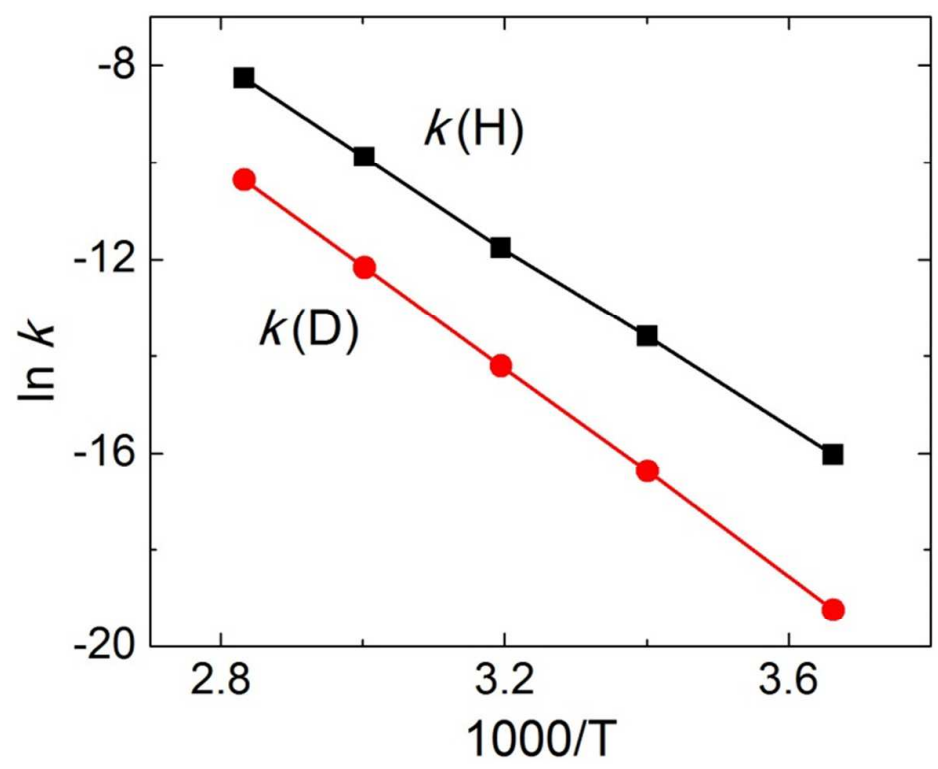

Figure S2. Arrhenius plot for the proton and deuterium transfer for the protonolysis of (COD)Pt ${ }^{\mathrm{II}} \mathrm{Me}_{2}$ by TFA via the concerted pathway at the M06/6-31G(d,p) level.

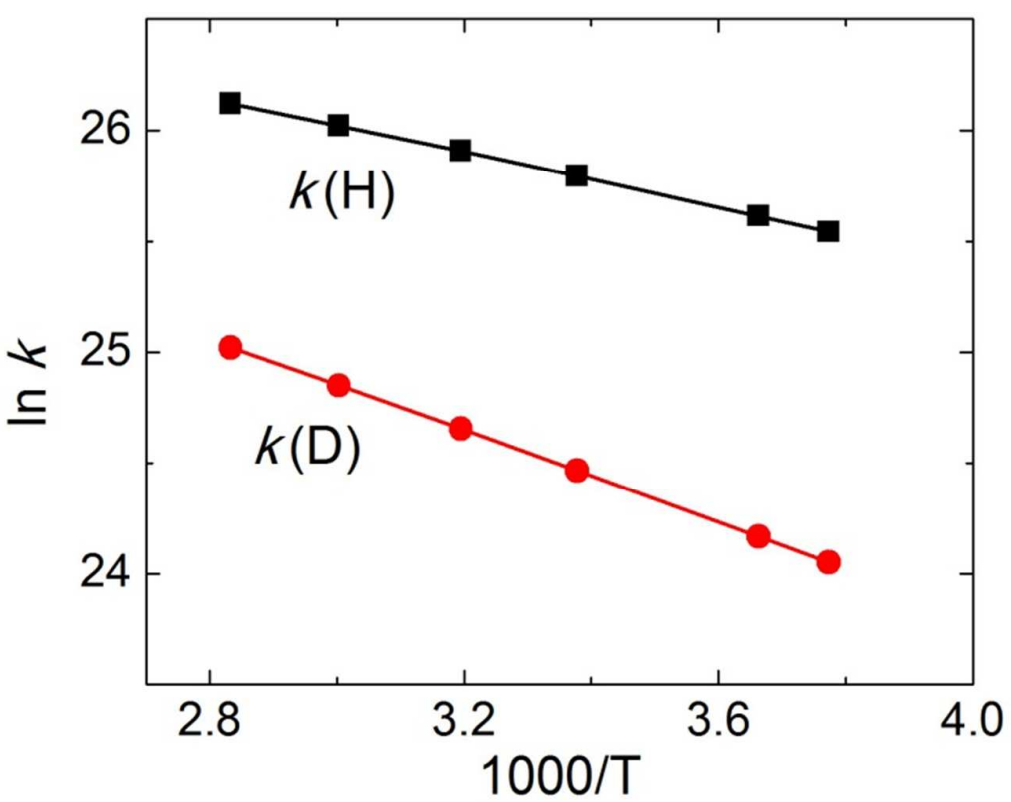

Figure S3. Arrhenius plot for the proton and deuterium transfer for the protonolysis of $\mathrm{ZnMe}_{2}$ by TFA via the concerted pathway at the M06/6-31G(d,p) level. 


\section{Detailed information for the VTST/MT method}

The VTST rate constants is given by ${ }^{3}$

$$
\begin{aligned}
k^{C V T} & =\min _{s} k^{G T}(T, s) \\
& =\sigma \frac{k_{B} T}{h} \frac{Q^{G T}\left(T, s_{*}^{C V T}\right)}{\Phi^{R}} \exp \left[-\beta V_{M E P}\left(s_{*}^{C V T}\right)\right]
\end{aligned}
$$

The superscript GT denotes the generalized transition state theory; $k_{B}$ is the Boltzmann constant; $h$ is Planck's constant; $S_{*}^{C V T}$ is the value of $s$ at which $k^{G T}$ is minimum, that is, the location of the canonical variational transition state; and $Q^{G T}$ and $Q^{R}$ are partition functions for the generalized transition state and reactants, respectively.

To include the tunneling effect, the calculated rate constant $k^{C V T}(T)$ is multiplied by a transmission coefficient, $\kappa^{S C T / G}$

$$
k^{C V T / G}(T)=\kappa^{C V T / G}(T) k^{C V T}(T)
$$

The transmission coefficient is defined as the ratio of the thermally averaged quantal transmission probability, $P^{G}(E)$, to the thermally averaged classical transmission probability for the effective vibrational adiabatic potential energy along the reaction coordinate that is implied by CVT theory, $P_{C}^{C V T / G}(E)^{4}$

$$
\kappa^{C V T / G}(T)=\frac{\int_{0}^{\infty} P^{G}(E) e^{-E / k_{B} T} \mathrm{~d} E}{\int_{0}^{\infty} P_{C}^{C V T / G}(E) e^{-E / k_{B} T} \mathrm{~d} E}
$$

The value of $P_{C}^{C V T / G}(E)$ is unity above the classical threshold energy and is zero below, this expression reduces to

$$
\kappa^{C V T / G}(T)=\frac{1}{k_{B} T} e^{V_{a}^{G}\left(s_{*}^{C V T}\right) / k_{B} T} \int_{0}^{\infty} P^{G}(E) e^{-E / k_{B} T} d E
$$

which can be written as 


$$
\kappa^{C V T / G}(T)=\frac{1}{k_{B} T} \kappa^{C V T / C A G} \int_{0}^{\infty}\left[P^{G}(E) e^{V^{A G} / k_{B} T}\right] e^{-E / k_{B} T} d E
$$

where

$$
\kappa^{C V T / C A G}=e^{\left[V_{a}^{G}\left(s_{*}^{C V T}\right)-V^{A G}\right] / k_{B} T}
$$

$V_{a}^{G}\left(s_{*}^{C V T}\right)$ is the ground-state adiabatic energy barrier evaluated at the canonical variational transition state, and $V^{A G}$ is defined by

$$
V^{A G}=\max _{s} V_{a}^{G}(s)
$$

The centrifugal-dominant small-curvature semiclassical adiabatic ground state (CDSCSAG) tunneling approximation ${ }^{5}$ was used to calculate $P^{G}(E)$. This method is called smallcurvature tunneling (SCT). The detailed mathematical derivations and computational formulas can be found elsewhere. ${ }^{6-8}$ 


\section{REFERENCES}

(1) Bercaw, J. E.; Chen, G. S.; Labinger, J. A.; Lin, B.-L., Organometallics 2010, 29, 4354-4359.

(2) Smith, D. C., Jr; Haar, C. M.; Stevens, E. D.; Nolan, S. P.; Marshall, W. J.; Moloy, K. G., Organometallics 2000, 19, 1427-1433.

(3) Garrett, B. C.; Joseph, T.; Truong, T. N.; Truhlar, D. G., Chem. Phys. 1989, 136, 271293.

(4) Garrett, B. C.; Truhlar, D. G.; Grev, R. S.; Magnuson, A. W., J. Phys. Chem. 1980, 84, 1730-1748.

(5) Liu, Y. P.; Lynch, G. C.; Truong, T. N.; Lu, D. H.; Truhlar, D. G.; Garrett, B. C., J. Am. Chem. Soc. 1993, 115, 2408-2415.

(6) Truhlar, D. G.; Isaacson, A. D.; Garrett, B. C., Generalized Transition State Theory. In Theory of Chemical Reaction Dynamics, Baer, M., Ed. CRC Press: Boca Raton, FL, 1985; Vol. 4, pp 65-137.

(7) Garrett, B. C.; Truhlar, D. G., Variational Transition State Theory. In Theory and Applications of Computational Chemistry: The First Forty Years, Dykstra, C. E.; Frenking, G.; Kim, K. S.; Scuseria, G. E., Eds. Elsevier: Amsterdam, 2005; pp 67-87.

(8) Fernandez-Ramos, A.; Ellingson, B. A.; Garrett, B. C.; Truhlar, D. G., Variational Transition State Theory with Multidimensional Tunneling. In Reviews in Computational Chemistry, Lipkowitz, K. B.; Cundari, T. R., Eds. Wiley-VCH: Hoboken, NJ, 2007; Vol. 23, pp 125-232. 